\section{Entlastung Mehrwert für Wundversorger}

\begin{abstract}
— Nach dem Motto "Sie versorgen die Patienten, wir versorgen Sie" möchte das Medizintechnik-Unternehmen OPED Wundversorger und Wundmanager noch stärker entlasten. Das Ziel: Mehr Zeit für die Patienten. Angesichts der geschätzt vier Millionen Menschen mit chronischen Wunden in Deutschland stehen Versorger von Wunden unter ständigem Zeitdruck. Auf Wunsch nimmt die bayerische Firma den Wundmanagern die gesamte Bürokratie und Logistik ab. Außerdem können alle handelsüblichen Produkte zur Versorgung chronischer Wunden und Hautpflege sowie Produkte zur Kompression erworben werden - per Telefon, Fax oder E-Mail. Die Artikel werden innerhalb 24 Stunden kostenlos an die gewünschte Adresse geschickt.
\end{abstract}

www.oped-wundversorgung.de

\section{Moderne Wundversorgung Neue Produktgrößen bieten mehr Komfort}

\begin{abstract}
— Flexiblen Halt und besseren Komfort verspricht Mepilex Border Flex jetzt in einer neuen Größe. Den ovalen Schaumverband mit Safetac Technologie gibt es seit August auch in 7,8 $\times 10 \mathrm{~cm}$. Er absorbiert Exsudat effektiv, hält es zurück und gewährleistet gleichzeitig ein feuchtes Wundmilieu. Die optimierte Form bietet eine bessere Anpassung an die Körperkonturen und ein Einrollen wird so weit wie möglich verhindert. Damit wird sowohl die Anpassungsfähigkeit als auch die Haftung verbessert. Mepilex Border Flex ist zur Behandlung von exsudierenden Wunden wie Dekubitalulzera, diabetischen Fußulzera, Bein- und Fußulzera, traumatischen Wunden und anderen sekundär heilenden Wunden vorgesehen. Optimal für den Einsatz auch an schwierigen Körperstellen. Auch Mepitel ${ }^{\circledR}$ One, eine transparente, einseitig mit Safetac Technologie beschichtete Wundauflage zur Behandlung schmerzhafter chronischer Wunden und Wunden mit empfindlicher Haut, ist jetzt flexibler nutzbar. Seit August ist die Wundauflage auf Rolle $(9,5 \times 150 \mathrm{~cm})$ und in Supersize 27,5 x 50 $\mathrm{cm}$ verfügbar, beispielsweise für die Versorgung von großfächigen Verbrennungen.
\end{abstract}

www.molnlycke.de

\title{
Monofilamentfaser-Pad: Wunden schnell und schmerzarm reinigen
}

— Die Entfernung von Belägen und Detritus ist in der Behandlung von Wunden essenziell. Für ein mechanisches Debridement hat sich die Wundreinigung mit Monofilamentfaser-Pads als effektiv erwiesen.

Etwa 99\% aller Mikroorganismen siedeln in Form von Biofilmen, also als mikrobielle Schleimfilme, die sich sowohl auf synthetischen, technischen Oberflächen, aber auch auf organischem, biologischen Material bilden können. Solche Biofilme kommen häufiger auf chronischen als auf akuten Wunden vor. Sie beeinflussen die Wundheilung negativ und tragen wesentlich zu Wundinfektionen bei. Damit stellen sie einen relevanten Faktor in der Entwicklung refraktärer Belagssituationen dar. Wichtig im Rahmen einer modernen Wundversorgung sei daher eine effektive Wundreinigung mit Entfernung von Belägen und Detri- tus, erklärte Dr. Thomas Eberlein, Gesamtärztlicher Leiter der Akademie ZWM Embrach (Schweiz) und Geschäftsführender Gesellschafter der WoundConsulting GmbH, Wien: „Das Debridement ist eine Grundvoraussetzung, um den funktionellen Prozess der Gewebsreparatur einzuleiten."

Die Möglichkeiten der Wundreinigung seien vielfältig, so Eberlein weiter. Das klassische chirurgische Debridement ist vor allem bei soliden Belägen und Nekrosen indiziert. Für das mechanische Debridement hat sich die Anwendung des Monofilamentfaser-Pads Debrisoft ${ }^{\oplus}$ als ein rasch und einfach anwendbares, effektives und schmerzarmes Verfahren erwiesen. Das Pad besteht aus mehr als 18 Millionen speziellen Mikrofasern auf einer Fläche von $10 \times 10 \mathrm{~cm}$, die die Wunde schneller und effizienter reinigen als Baumwollgewebe. Die Faserspitzen lösen
Debris und Exsudat aktiv aus der Wunde, die dann im Faserverbund aufgenommen werden. So wird bei kurzer Behandlungszeit und einfachem Handling eine saubere Wunde geschaffen, die mittels phasengerechter Wundauflage oder Unterdrucktherapie weiter versorgt werden kann. Die positiven Eigenschaften des Monofilamentfaser-Pads konnten in einer multizentrischen prospektiven Observationsstudie belegt werden, in der sich eine hohe Anwenderzufriedenheit [Bahr S et al. J Wound Care 2011; 20(5): 242-8] zeigte.

(koc)

Symposium „Wundversorgung im Alltag schmerzarm und effektiv", Deutscher Wundkongress und Bremer Pflegekongress,

Bremen, 7. Mai 2015 (Veranstalter: Lohmann \& Rauscher GmbH \& Co. KG, Neuwied) 